\title{
A retrospective cohort study of hemostatic agent use during hysterectomy and risk of post-operative complications
}

\author{
John A. Harris ${ }^{1 *}$ | Shitanshu Uppal ${ }^{2}$ | Neil Kamdar ${ }^{3}$ | Carolyn W. Swenson ${ }^{4}$ | \\ Darrell Campbell $^{5}$ | Daniel M. Morgan ${ }^{4}$
}

\begin{abstract}
${ }^{1}$ Division of Women's Health, Department of Obstetrics and Gynecology, University of Michigan, Ann Arbor, MI, USA

${ }^{2}$ Division of Gynecologic Oncology, Department of Obstetrics and Gynecology, University of Michigan, Ann Arbor, MI, USA

${ }^{3}$ Department of Obstetrics and Gynecology, University of Michigan, Ann Arbor, MI, USA

${ }^{4}$ Division of Urogynecology, Department of Obstetrics and Gynecology, University of Michigan, Ann Arbor, MI, USA

${ }^{5}$ Department of Surgery, University of Michigan, Ann Arbor, MI, USA

${ }^{*}$ Correspondence

John A. Harris, Division of Women's Health, Department of Obstetrics and Gynecology, University of Michigan, Ann Arbor, MI, USA. Email: harrisja@mail.magee.edu
\end{abstract}

\section{Funding Information}

Blue Cross and Blue Shield of Michigan/ Blue Care Network; Robert Wood Johnson Foundation; National Institute of Child Health and Human Development Women's Reproductive Health Research Career Development

\begin{abstract}
Objective: To determine if the use of intraoperative hemostatic agents was a risk factor for post-operative adverse events within 30 days of patients undergoing hysterectomy.
\end{abstract}

Method: A population-based retrospective cohort study included data from patients undergoing hysterectomy for any indication between January 1, 2013, and December 31, 2014, at 52 hospitals in Michigan, USA. Any individuals with missing covariate data were excluded, and multivariable logistic regression and propensity score-matching were used to estimate the rate of post-operative adverse events associated with intraoperative hemostatic agents independent of demographic and surgical factors.

Results: There were 17960 surgical procedures included in the analysis, with 4659 (25.9\%) that included the use of hemostatic agents. Hemostatic agent use was associated with an increase in predicted hospital re-admissions $(P=0.007)$. Among all hysterectomy approaches, and after adjusting for demographic and surgical factors, hemostatic agent use during robotic-assisted laparoscopic hysterectomy was associated with an increased predicted rate of blood transfusions $(P=0.019)$, an increased predicted rate of pelvic abscess diagnoses $(P=0.001)$, an increased predicted rate of hospital re-admission $(P=0.001)$, and an increased predicted rate of re-operation $(P=0.021)$.

Conclusion: Hemostatic agents should be used carefully owing to associations with increased post-operative re-admissions and re-operations when used during hysterectomy.

\section{KEYWORDS}

Blood transfusions; Complications; Hemostatic agents; Hospital readmissions; Hysterectomy; Pelvic abscess; Propensity score matching

\section{1 | INTRODUCTION}

Excessive bleeding during surgery, and subsequent need for blood transfusion, is one of the commonest major adverse events across all surgeries. ${ }^{1}$ In the management of intra-operative bleeding, hemostasis can be achieved using manual pressure, suture ligation, electrocautery, ultrasonic coagulation, laser ablation, staples and clips, or the application of hemostatic products. Combinations of these are often employed in surgery; however, there is limited knowledge regarding the comparative effectiveness of these methods across every surgical procedure.

Hemostatic agents have been used widely in surgery and their use is increasing; of 430000 hysterectomies performed in the USA in 2010, hemostatic agents were utilized in $14 \%$ of procedures-approximately 
60000 hysterectomies. ${ }^{2,3}$ However, there is a paucity of data supporting the routine use of hemostatic agents during hysterectomy to reduce the need for post-operative transfusions beyond traditional mechanical and electric-cautery techniques. Further, these agents add costs to each procedure and could be associated with adverse events requiring hospital re-admission, antibiotic administration, and re-operation. $^{4-11}$

Therefore, the aim of the present study was to estimate, among patients undergoing hysterectomy, the effect of hemostatic agents on post-operative blood transfusion rates, pelvic abscess diagnoses, hospital re-admissions, and re-operations.

\section{2 | MATERIALS AND METHODS}

A retrospective cohort study included data from the Michigan Surgical Quality Collaborative (MSQC) database, a large, observational, multi-center clinical database of surgical and post-operative care in Michigan, USA. Data in the MSQC database from patients who underwent a hysterectomy for any indication between January 1, 2013 and December 31, 2014 were included in the study; patient data were excluded if there were any missing covariate data. The study included de-identified patient records and the University of Michigan institutional review board granted "Not Regulated" status to the study (HUM00073978).

The MSQC is a Blue Cross Blue Shield of Michigan/Blue Care Network-funded database voluntarily populated by both academic and community hospitals throughout Michigan, USA. At the time the present study was conducted, data from 52 participating hospitals were available for analyses. At each site, data were abstracted from medical records by trained nurse abstractors. Patient characteristics, intra-operative processes of care, and 30-day post-operative outcomes from hysterectomy procedures at contributing hospitals were routinely collected. To reduce sampling error, a standardized data collection methodology was employed using only the first 25 surgical procedures of an 8-day cycle. Detailed methods of the registry's data collection have been described previously. ${ }^{12,13}$

The primary outcome variable was the presence of the major post-operative adverse events blood transfusion, diagnosis of pelvic abscess, hospital re-admission, and re-operation within 30 days of initial surgery. Post-operative blood transfusion was defined as the transfusion of any number of packed red blood cells after the primary surgery. Post-operative hospital readmission was defined as an inpatient hospital re-admission. Pelvic abscess diagnosis was defined as a surgical site infection in the organ space recorded in the patient's medical records. Post-operative re-operation was defined as any postoperative surgical operation for any indication, other than the completion of cancer staging.

The primary independent variable was the use of a hemostatic agent (oxidized cellulose polymer, thrombin-containing gelatin matrix, absorbable gelatin compressed sponge, or hemostatic agent of unknown type), as recorded in the operative notes. Owing to the overlap in the usage of these products and the high rate of hemostatic agent usage without specifically identifying the agent, associations between individual products and major post-operative adverse events were not analyzed. Patients were only identified as having received of not received a hemostatic agent.

The patient and surgical characteristics include in the analyses as covariates were age, race, body mass index, surgical indication (cancer, uterine fibroids, or other), surgical approach (abdominal, laparoscopic non-robotic-assisted, robotic-assisted, or vaginal), estimated blood loss, measured uterine specimen weight, and surgical time. To help control for confounding by the complexity of surgical procedures, the total relative value units (based on current procedural terminology codes) were calculated for the total concurrent operative procedures. Finally, overall patient comorbidity was measured using a modified Charlson comorbidity score. ${ }^{14}$

Descriptive statistics were analyzed using the $\chi^{2}$ test for categorical variables and confidence intervals (Cls) were calculated using the Wilson score interval for binomial distributions. Multivariable logistic regression including the covariates described above and propensity score matching was performed to isolate associations between hemostatic agent use and post-operative adverse events. The regression model included an interaction term between hemostatic agent use and surgical approach. All variables were analyzed as continuous variables except for surgical indication and approach, which were analyzed as categorical variables. The propensity score was calculated using the covariates detailed above.

The matched cohort included a 1:2 ratio between patients who received hemostatic agents and those who did not; a 0.1-unit caliper width was used as a replacement. Average effects were reported in terms of predicted outcome probabilities with the presence or absence of hemostatic agents, with all other covariates maintained at their observed values because effect sizes could not be inferred directly from coefficients or odds ratios of logit models. Two-tailed hypothesis testing was performed and $P<0.05$ was considered statistically significant. STATA version 13.1 (StataCorp LP, College Station, Texas, USA) was used for all analyses.

\section{3 | RESULTS}

There were 18302 hysterectomies recorded during the study period; 202 (1.1\%) were excluded because the surgeries were not performed by a gynecologist and $140(0.8 \%)$ were excluded owing to incomplete covariate data. Consequently, the full study cohort included 13301 patients who did not receive hemostatic agents and 4659 who did (Fig. 1). There were 13974 patients included in the propensity score matched cohort, including 9316 (66.7\%) patients who did not receive a hemostatic agent and 4658 (33.3\%) patients who did. Among the full study cohort, patients who received hemostatic agents were more likely have fibroids $(P<0.001)$, to have undergone robotic surgery $(P<0.001)$, and to experience an estimated blood loss of at least $250 \mathrm{~mL}(P<0.001)$. After propensity score matching, the cohort was found to be closely matched across all characteristics (Table 1). 


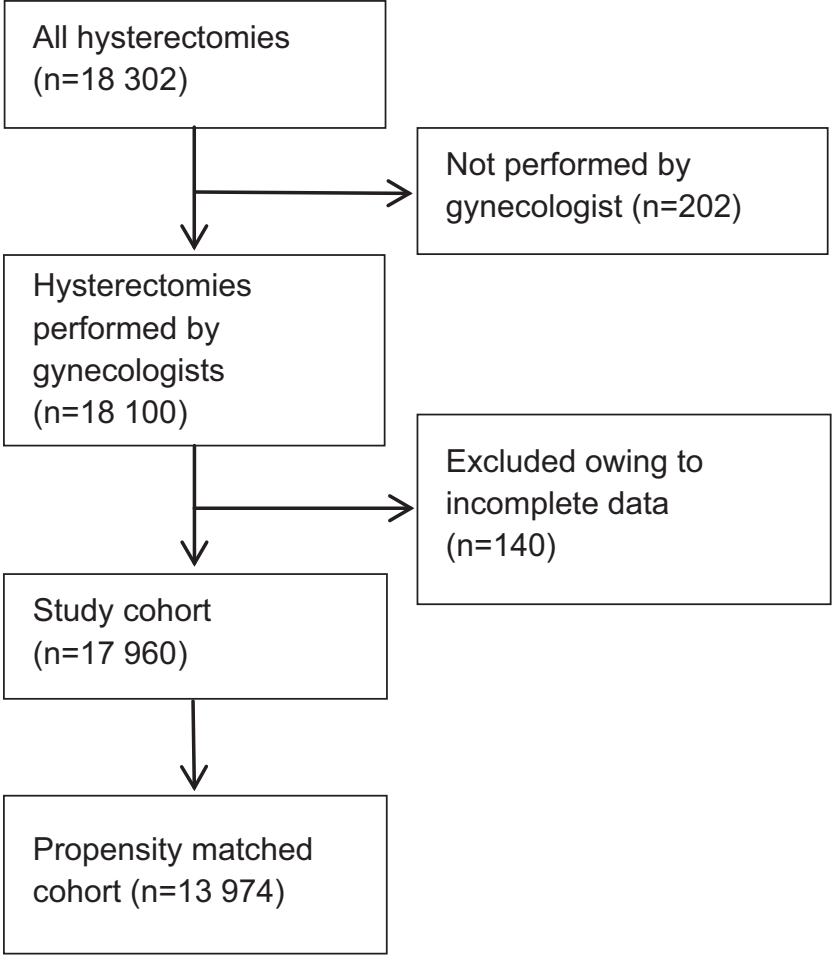

FIGURE 1 Flow diagram of patient selection.

The overall prevalence of post-operative blood transfusion was 2.7\% (95\% Cl 2.5-3.0). The logistic regression model demonstrated no association between the use of hemostatic agents and the predicted prevalence of blood transfusion $(P=0.291)$ (Table 2). This was consistent with the estimate from the propensity score matched cohort $(P=0.764)$.

The overall prevalence of pelvic abscess was $1.0 \%(95 \% \mathrm{Cl} 0.9-$ 1.2) and no association was observed between hemostatic agent use and the incidence of pelvic abscesses in either the logistic regression variable model $(P=0.144)$ or the propensity score matched cohort $(P=0.188)$ analyses.

The rate of hospital re-admissions across the complete cohort was 4.0\% (95\% Cl 3.7-4.3). The most common re-admission diagnoses were related to infection and hematoma. Both the logistic regression variable model $(P=0.047)$ and the propensity score matched cohort $(P=0.007)$ found that the use of hemostatic agents was associated with increases in post-operative hospital re-admission.

Re-operations were performed for $2.1 \%$ ( $95 \% \mathrm{Cl} 1.9-2.3$ ) of surgical patients during the study period. The most common surgical indication for re-operation was hemorrhage or hematoma. No association was observed between the application of hemostatic agents and requiring post-operative re-operation in the logistic regression variable model $(P=0.075)$ or propensity score matched cohort $(P=0.090)$.

The outcomes of interest were also analyzed in terms of the surgical approach used during hysterectomy. Among surgeries performed using vaginal and abdominal approaches, no differences were observed in transfusions, pelvic abscesses, re-admissions, or re-operations between patients who received hemostatic agents and those who did not. The use of hemostatic agents during robotic-assisted laparoscopic procedures was associated with a greater incidence of predicted blood transfusions $(P=0.019)$, pelvic abscess diagnoses $(P=0.001)$, hospital re-admissions ( $P=0.001)$, and re-operations ( $P=0.021$ ) (Table 3$)$. In laparoscopic hysterectomy procedures, the use of hemostatic agents was associated with an increase in predicted re-admissions $(P=0.045)$ and predicted re-operations $(P=0.046)$.

\section{4 | DISCUSSION}

The present study confirmed previous findings of associations between hemostatic agents and post-operative adverse events among patients who have undergone hysterectomies. In this retrospective study that examined hysterectomies performed for any indication from a statewide database in the USA, no benefit was observed for the use of hemostatic agents in terms of reduced blood transfusions, and increased risks for hospital re-admission and re-operation were recorded when hemostatic agents were used.

Hemostatic agents are designed to improve intra-operative and post-operative hemostasis, decreasing surgical and post-surgical adverse events such as requiring blood transfusions, the formation of post-operative hematomas, and re-operations. ${ }^{15-19}$ In the present cohort, they were used in approximately $26 \%$ of all hysterectomy procedures. This high rate of use suggests that they are used both to achieve hemostasis and as a prophylactic measure. Further, this high rate of utilization should be scrutinized in terms of evidence of a specific need in addition to evidence of an absence of harm. Previous studies supporting these benefits in hysterectomy are lacking, ${ }^{4-11}$ and the present analysis, confirming findings of previous studies, identified that these agents could be harmful.

There are multiple possible reasons why hemostatic agents could be associated with post-operative adverse events of hysterectomies. Hemostatic agents could provide inferior hemostasis in the hours and days following surgery compared with traditional hemostatic methods. Increased post-operative bleeding, even in small amounts, could lead to appreciable hematomas, in turn increasing the risk for pain through mass effects or inflammatory cytokines, or for infection by acting as a medium for the growth of bacteria; all of these could result in emergency department visits and re-admission. ${ }^{1}$ Further, the presence of these foreign materials could cause persistent fluid collection that eventually becomes symptomatic, leading to re-admission. ${ }^{6-11}$ Hospital re-admissions are used as a target for quality improvements and reimbursement oversight. ${ }^{20,21}$ Hospital re-admission events cost Medicare approximately US $\$ 17.4$ billion in 2004 and have been a target of performance-based repayment by the agency. ${ }^{20,21}$ These re-admissions and re-operations are an important, potentially avoidable outcome. Further, these findings are consistent with, and a reasonable extension of, previous studies of hemostatic agents. ${ }^{4-11}$

Previous studies in smaller cohorts have reported similar findings regarding associations between hemostatic agents and pelvic abscesses. ${ }^{4,5}$ Anderson et al. ${ }^{5}$ found an increased risk of pelvic abscess after the use of a gelatin-thrombin matrix, as well as with 
TABLE 1 Baseline characteristics of patients undergoing hysterectomies ( $n=17960$ ). ${ }^{\text {a }}$

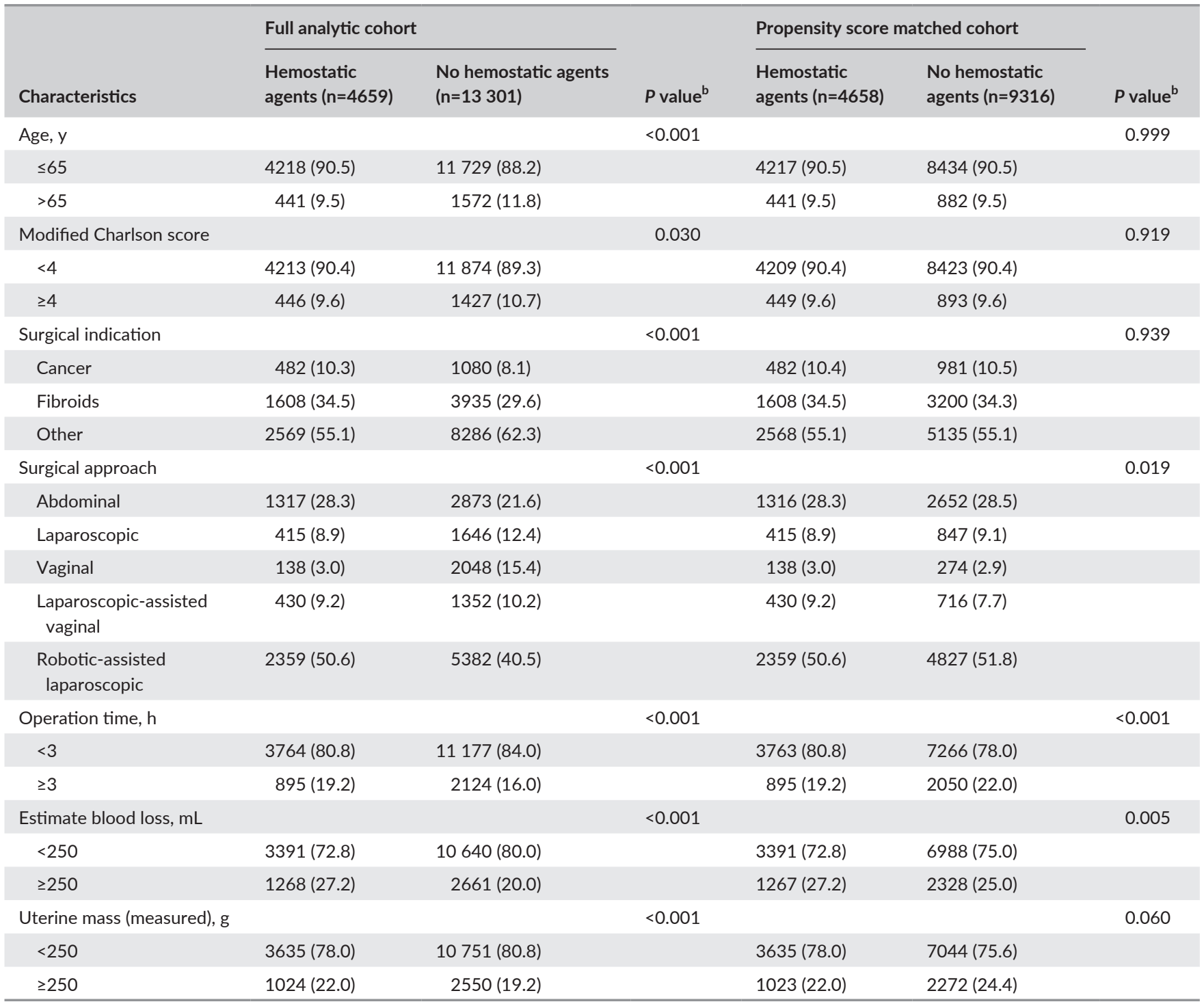

${ }^{a}$ Values are given as number (percentage) unless indicated otherwise.

${ }^{b} \chi^{2}$ test.

oxidized regenerated cellulose. In the present study, the use of hemostatic agents was only associated with the diagnosis of pelvic abscess in robotic-assisted hysterectomies. This could result from a reliance on bipolar cautery and hemostatic agents to control surgical bleeding, compared with a variety of methods used during traditional hysterectomies. Additionally, the robotic-assisted hysterectomy data had the greatest statistical power and consequently, was better placed to detect a statistically significant result compared with the other analyses of individual surgical approaches. Of note, the causes for re-admission in the present cohort were frequently fever and infection. These indications for admission could be due to other infectious etiology, such as urinary tract infections, pneumonia, or superficial surgical site infections; however, it is also possible that these more general billing codes could actually represent pelvic abscess symptoms. Owing to this, pelvic abscess could be a systematically under-attributed cause of re-admission in billing or nurse-abstracted data.
Hemostatic agents are used widely in gynecologic surgery and their use is increasing. ${ }^{3}$ In addition to their lack of proven benefit, these agents are expensive. In a report from one academic center in $2014,{ }^{15}$ the cost was identified as being between US $\$ 60$ and $\$ 972$ per agent per surgery. Assessing the benefits and harms of using hemostatic agents is important to patients, surgeons, and payers. Further, the costs of re-admission significantly increase the total costs that could be attributed to the use of these agents.

There were limitations to the present study. Even when multivariable regression and propensity score matching is used, it is susceptible to unmeasured confounder bias, as measured and unmeasured confounding can cause overestimation of effect sizes. Known confounders were tested and controlled for but there could have been other unmeasured confounders that were not adjusted for, including surgeon experience, skill, or measures of hospital quality, that could have been associated with hospital-level use of hemostatic agents. The analyses attempted to control for the surgical difficulty of the procedures by 
TABLE 2 Estimated incidence of post-operative adverse events.

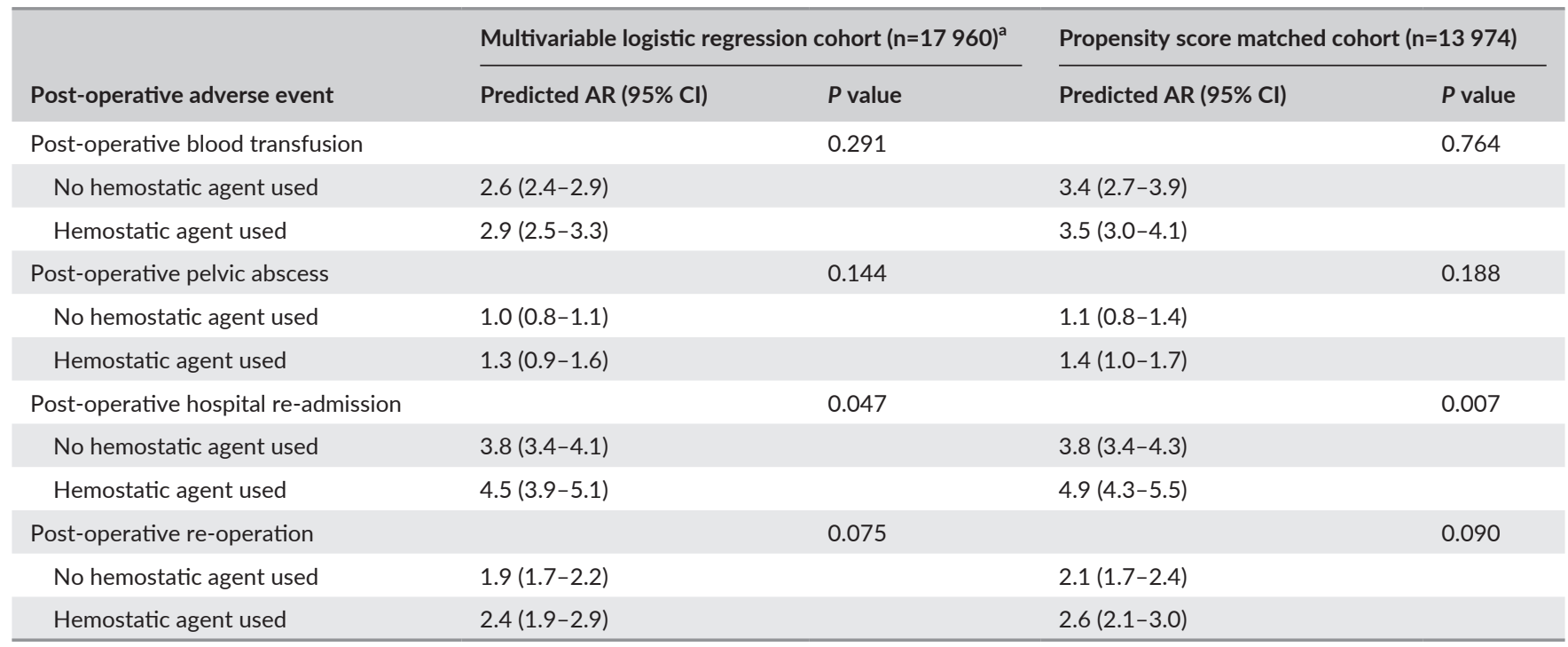

Abbreviations: $\mathrm{AR}$, absolute rate as a percentage; $\mathrm{Cl}$, confidence interval.

${ }^{a}$ The confounding variables included in the model were age, race, modified Charlson score, body mass index, surgical indication, surgical approach, concurrent procedure relative value units, estimated surgical blood loss, measured uterine specimen weight, and operative length.

evaluating concurrent procedures being performed, operative time, blood loss, and uterine mass; however, this is not a completely comprehensive collection of all the factors that comprise surgical complexity. Additionally, a key limitation of the present study was that it was formed from a sample of Michigan community and university hospitals. It is not possible to rule out geographic variations in usage and this could limit the generalizability of the findings to other regions of the USA. Further, the exact type of hemostatic agent used was not well described in the data so it was not possible to break down the effects of individual products. Finally, data abstraction from operative reports could have underestimated the rate of hemostatic agent use and did not include information regarding important events related to the surgery that occurred later than 30 days after hysterectomy or any undocumented events that occurred outside of the primary hospital.

The present study found that the utilization of hemostatic agents at the time of hysterectomy was a risk factor for re-admissions and re-operations within 30 days of surgery in robotic and laparoscopic hysterectomies. The use of surgical technology should be based on the rigorous study of patient-centered outcomes, even when the technology has been in use for a long period of time. In the absence of data

TABLE 3 Estimated incidence of post-operative adverse events in the propensity score cohort among different surgical approaches.

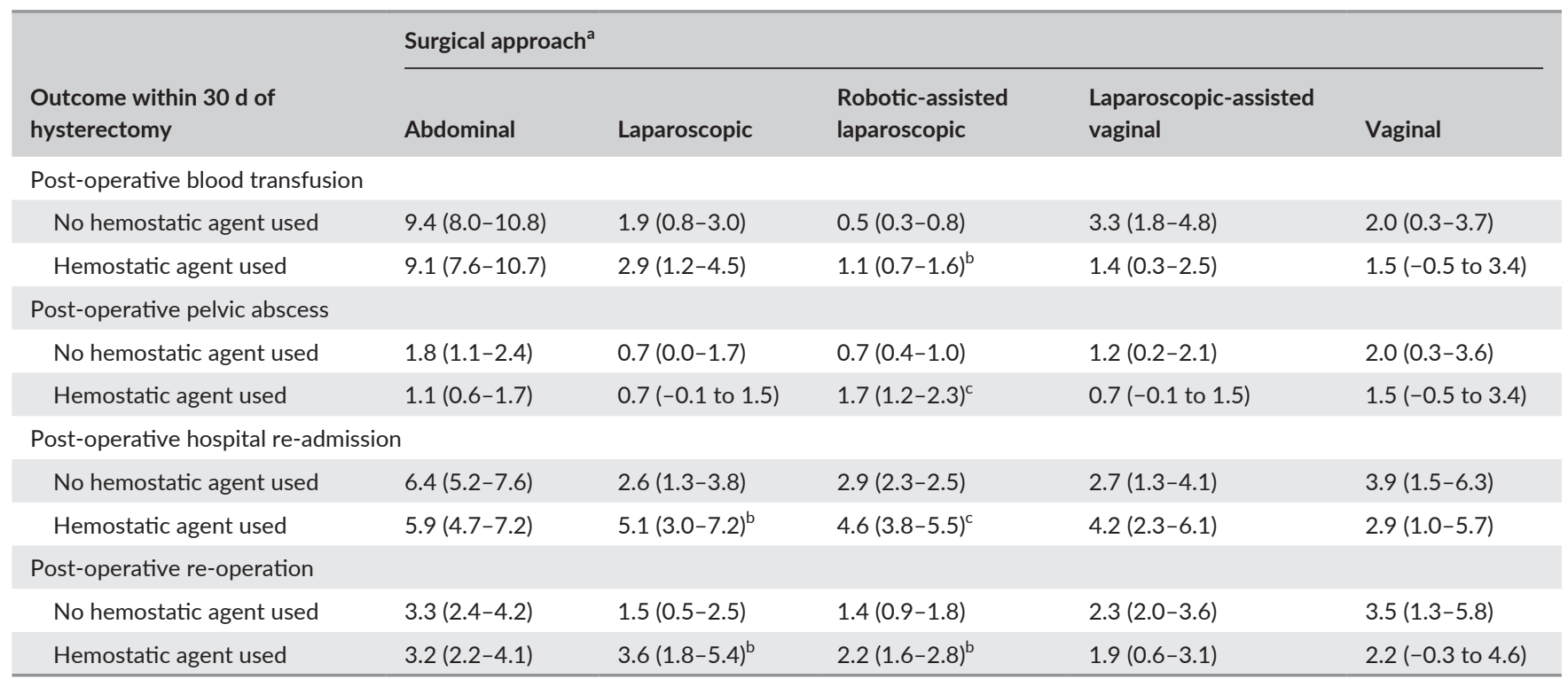

${ }^{a}$ Values are given as predicted absolute rate ( $95 \%$ confidence interval).

${ }^{\mathrm{b}} P<0.050$.

${ }^{\mathrm{c}} P \leq 0.001$. 
demonstrating a clear benefit to their use in hysterectomy, hemostatic agents should be used judiciously given the possible increased risk of post-operative adverse events.

\section{AUTHOR CONTRIBUTIONS}

$\mathrm{JAH}, \mathrm{SU}, \mathrm{NK}, \mathrm{CWS}$, and DMM conceived and designed the study. $\mathrm{JAH}, \mathrm{DC}$, and DMM retrieved the data. JAH and NK undertook the statistical analyses. JAH and SU drafted the article. All authors revised and approved the final report.

\section{ACKNOWLEDGMENTS}

Michigan Surgical Quality Collaborative database is funded by Blue Cross and Blue Shield of Michigan/Blue Care Network. JAH is a Robert Wood Johnson Foundation Clinical Scholar at the University of Michigan. The Robert Wood Johnson Foundation and Blue Cross and Blue Shield of Michigan/Blue Care Network were not directly involved in study design, data acquisition and interpretation, manuscript preparation, or review. Any opinions expressed herein do not necessarily reflect the opinions of the Robert Wood Johnson Foundation. Investigator support for JAH was provided by the National Institute of Child Health and Human Development Women's Reproductive Health Research Career Development Award K12 HD063087. Investigator support for CWS was provided by the National Institute of Child Health and Human Development Women's Reproductive Health Research Career Development Award K12 HD065257.

\section{CONFLICT OF INTEREST}

The authors have no conflicts of interest.

\section{REFERENCES}

1. Dessources K, Hou JY, Tergas Al, et al. Factors associated with 30-day hospital readmission after hysterectomy. Obstet Gynecol. 2015;125:461-470.

2. Wright JD, Herzog TJ, Tsui J, et al. Nationwide trends in the performance of inpatient hysterectomy in the United States. Obstet Gynecol. 2013;122(2Pt1):233-241.

3. Wright JD, Ananth CV, Lewin SN, etal. Patterns of use of hemostatic agents in patients undergoing major surgery. J Surg Res. 2014;186:458-466.
4. Fagotti A, Costantini B, Fanfani F, et al. Risk of postoperative pelvic abscess in major gynecologic oncology surgery: One-year singleinstitution experience. Ann Surg Oncol. 2010;17:2452-2458.

5. Anderson CK, Medlin E, Ferriss AF, et al. Association between gelatinthrombin matrix use and abscesses in women undergoing pelvic surgery. Obstet Gynecol. 2014;124:589-595.

6. Behbehani S, Tulandi T. Oxidized regenerated cellulose imitating pelvic abscess. Obstet Gynecol. 2013;121:447-449.

7. Nakajima M, Kamei T, Tomimatu K, Manabe T. An intraperitoneal tumorous mass caused by granulomas of microfibrillar collagen hemostat (Avitene). Arch Pathol Lab Med. 1995;119:1161-1163.

8. Suzuki Y, Vellinga TT, Istre O, Einarsson Jl. Small bowel obstruction associated with use of a gelatin-thrombin matrix sealant (FloSeal) after laparoscopic gynecologic surgery. J Minim Invasive Gynecol. 2010;17:641-645.

9. Hobday CD, Milam MR, Milam RA, Euscher E, Brown J. Postoperative small bowel obstruction associated with use of hemostatic agents. J Minim Invasive Gynecol. 2009;16:224-226.

10. Carter RM, Halliwell B, Harkins G. Pelvic inflammatory reactions to Floseal. J Minim Invasive Gynecol. 2011;18:284.

11. Clapp B, Santillan A. Small bowel obstruction after FloSeal use. JSLS. 2011:15:361.

12. Mahnert N, Morgan D, Campbell D, Johnston C, As-Sanie S. Unexpected gynecologic malignancy diagnosed after hysterectomy performed for benign indications. Obstet Gynecol. 2015;125: 397-405.

13. Corona LE, Swenson CW, Sheetz KH, et al. Use of other treatments before hysterectomy for benign conditions in a statewide hospital collaborative. Am J Obstet Gynecol. 2015;212:304.e1-304.e7.

14. Charlson ME, Pompei P, Ales KL, MacKenzie CR. A new method of classifying prognostic comorbidity in longitudinal studies: Development and validation. J Chronic Dis. 1987;40:373-383.

15. Wysham WZ, Roque DR, Soper JT. Use of topical hemostatic agents in gynecologic surgery. Obstet Gynecol Surv. 2014;69:557-563.

16. Echave M, Oyaguez I, Casado MA. Use of Floseal(R), a human gelatinethrombin matrix sealant, in surgery: A systematic review. BMC Surg. 2014;14:111.

17. Oz MC, Rondinone JF, Shargill NS. FloSeal Matrix: New generation topical hemostatic sealant. J Card Surg. 2003;18:486-493.

18. Oz MC, Cosgrove 3rd DM, Badduke BR, et al. Controlled clinical trial of a novel hemostatic agent in cardiac surgery. The Fusion Matrix Study Group. Ann Thorac Surg. 2000;69:1376-1382.

19. Barnes AC. The use of gelatin foam sponges in obstetrics and gynecology. Am J Obstet Gynecol. 1947;54:105-107.

20. Jencks SF, Williams MV. Coleman Ea: Rehospitalizations among patients in the Medicare fee-for-service program. $N$ Engl J Med. 2009:360:1418-1428.

21. Tsai TC, Joynt KE, Orav EJ, Gawande AA, Jha AK. Variation in surgical-readmission rates and quality of hospital care. $N$ Engl J Med. 2013:369:1134-1142. 